also found in another family outbreak with secondary and tertiary cases (including one with Stevens-Johnson syndrome). ${ }^{8}$ This interval is longer than that estimated by W. S. Jordan ${ }^{9}$ for atypical pneumonia or observed in experimental infections with $M$. pneumoniae. ${ }^{10}$ However, Jordan's report was made before specific diagnostic tests for the detection of $M$. pneumoniae were available, and infections due to other respiratory pathogens may have complicated the results. In experimental infections the relatively large doses of $M$. pneumoniae administered probably resulted in a shorter incubation period than that following natural infection. A relatively long incubation period is consistent with the slow spread previously noted for $M$. pneumoniae infection. ${ }^{1}$

Patients excreted $M$. pneumoniae over long periods. Nearly half of them yielded positive throat swabs after two months, and the organism was still present in five patients 12-13 weeks after onset of the disease. Treatment with tetracyclines did not necessarily eliminate the organism from the throat, nor did it reduce the incidence of prolonged cough. It is not known whether the organism is transmissible during these prolonged periods of excretion or persistent cough or only during the early stages of infection.

Clinically the disease ranged from pneumonia or pneumonitis to an asymptomatic infection. Most cases were mild and diagnosed as non-specific respiratory illness. But some evidence of infection in the lower respiratory tract was noted in $71 \%$ of the infected contacts. Ear infection occurred in $23 \%$ of 87 patients with respiratory illness.

The reports from Seattle ${ }^{348}$ are a valuable contribution to the epidemiology of $M$. pneumoniae infection in a civilian population. Similar studies are needed elsewhere to determine whether the spread of $M$. pneumoniae follows the same pattern. Decisions on the potential value of $M$. pneumoniae vaccines in civilian populations will rest on such information.

\section{Psychiatric Rehabilitation}

Whilst traditional forms of occupational therapy have played a part in psychiatric rehabilitation for many years, it is only recently that interest has been aroused in methods of applying industrial therapy in hospital. Such work often includes minor manipulative procedures, and, being mainly by subcontract to outside firms, is comparable to normal factory activity. Mamufacture of ball-point pens, toys, cardboard boxes, and cement blocks is some of the work being done. In 1958 half the montal hospitals used this type of therapy, ${ }^{1}$ and today it would be difficult to find a hospital without some

\footnotetext{
' Charlton, E. P. H., in Proceedings of a Conference on the Place of Work in the Treatment of Mental Disorder, p. 29, 1959. National - Association for Mental Health, London.

Brit. med. F., 1966, 1, 1100.

- Macmillan, D., Lancet, 1958, 2, 201.

- Howells, J. G., Family Psychiatry, 1963. Edinburgh and London. tation of Long-stay Schizophrenic Patients. Industrial RehabiliCouncil Memorandum No. 42, 1964. H.M.S.O., London.

Jones, M., Lancet, 1956, 2, 985.

111, 955

Annual Reports, 1961-5, Industrial Therapy Organization (Bristol) Ltd. Bristol.

Brit. med. 7., 1966, 1, 1532.

Ibid., 1966, 2, 655.

Barton, R., Institutional Neurosis, 2nd ed., 1966. Bristol.
}

form of it. Visitors to the B.M.A.'s Annual Clinical Meeting at Worcester this year saw an interesting demonstration of arrangements in the Birmingham Region. ${ }^{2}$

The need for occupation was recognized by Pinel and Tuke in the late eighteenth century, when the liberalization of mental treatment began. The "continuous process of translating the disabled by full medical care to being producers and earners," as envisaged by Beveridge, represented modern thinking but failed to emphasize the special problems of social resettlement of the mentally ill. The habit-training programmes for deteriorated schizophrenics confined to institutions played their part, and the process continued with work training. Therapeutic communities, by deliberate simulation of natural conditions, permitted social reintegration of patients within the hospital environment, and the concept of community care now embodied in the Mental Health Act (1959) provided the framework for social and personal reinstatement. A comprehensively organized mental health service for the local community as in Nottingham, ${ }^{3}$ and the principles underlying organized family psychiatry in Norwich, ${ }^{4}$ have wide prophylactic implications.

The importance of industrial therapy in psychiatric hospitals is undoubted but difficult to estimate precisely. Yardsticks are elusive. J. K. Wing and colleagues ${ }^{5}$ mention that repeated practice alters the results, and that by the crude measure of workshop output the performance of severely ill chronic schizophrenics varied with changes in supervision. They also pointed out the artificiality of distinguishing between long- and short-stay patients. But they showed that 21 out of 45 moderately disabled schizophrenics were working satisfactorily a year after discharge from an industrial rehabilitation unit. Acceptance of psychiatric patients at Ministry of Labour industrial rehabilitation units is customary now, and figures for rehabilitation suggest results comparable with those of other disabled groups. ${ }^{6}$ Selection of patients is clearly an important factor, and it has been suggested also that too high a proportion of psychiatric patients in these units defeats the object of them.

Important advances in the provision of sheltered employment for the mentally ill have been made in recent years. From 1961 St. Wulstan's Hospital, Malvern, previously a sanatorium, has been used for rehabilitation of long-stay patients drawn from the twelve psychiatric hospitals in the Birmingham region. A therapeutic community is provided in combination with financial arrangements which include incentives and deterrents as well as deductions at source from patients' wages for sickness benefit and for a resettlement grant on discharge. ${ }^{7}$ Industrial therapy organizations operating as commercial concerns have also been set up. Bristol led the field from 1959, and the management of its organization includes representatives from industry, civic authorities, the Church, and trade unions. This scheme has been approved by the Ministry of Labour. ${ }^{8}$ The enterprise at Bristol has been followed by other industrial therapy organizations in Surrey, Middlesex, Cheshire, and Northern Ireland. In 1963 Birmingham formed an industrial therapy association, began its activities with a car-washing unit (as at Bristol), and has now opened a factory, now also recognized by the Ministry of Labour. The early approval of the workshop at Bristol has now been extended by the Ministry to a sheltered placements scheme for selected patients in industry. Bristol has also recently opened a petrol-filling station manned by psychiatric patients. At Cheadle Royal Hospital an industrial therapy unit now approved by the Ministry has been set up with the assistance of grants from the Nuffield 
Foundation, ${ }^{9}$ and the Manchester Regional Hospital Board has recently contracted for patients from regional hospitals to work in this unit, which has specialized in certain products.

Many regional hospital boards are considering setting up schemes of industrial work therapy, and an encouraging prospect of collaboration with local health authorities exists. Further provision of hostels by the latter will, it is hoped, progress in parallel. In Birmingham the industrial therapy association works in close liaison with the Association for Mental Health, and this body has recently opened a hostel close to the factory. Through these occupational organizations community care may become more effective and acceptable. ${ }^{10}$ Employers have contact with patients in their working conditions within the hospitals. And the general public have points of contact (though so far in restricted numbers) with the patients through car-washing services, which produce immediate results-among them a satisfied customer. Increased acceptance of industrial therapy will help to banish the old institutional neurosis for ever. ${ }^{11}$

\section{Cotton-wool Spots}

Some few years after von Helmholtz had devised the ophthalmoscope Richard Liebreich, chief assistant to A. von Graefe, described " rounded, bright, milk-white, slightly raised spots" which he had observed with the ophthalmoscope in patients with Bright's disease. ${ }^{1}$

Because of their fluffy appearance, the retinal spots which Liebreich had described became known as cotton-wool spots. They are usually restricted to the posterior segment of the fundus, they rarely exceed one-third of the size of the optic disc, and disappear without trace in six to twelve weeks. They are found in diseases associated with arterial hypertension and also in a number of diseases of arterioles such as polyarteritis nodosa and systemic lupus erythematosus, as a result of blocking of these vessels in fat embolism and subacute bacterial endocarditis, and also in severe anaemias such as pernicious anaemia and anaemia after haemorrhage. Histological study of cotton-wool spots shows them to contain characteristic clusters of "cytoid bodies" in the nerve fibre layer. These are round or spindle-shaped cell-like structures with granular pseudocytoplasm and eosinophilic nucleus-like globules.

When J. S. Friedenwald found it possible to visualize the retinal vascular network by staining the retina with Schiff's reagent he discovered that the cytoid bodies also contained material which stained intensely by this technique. The cotton-wool spots were regularly located within the terminal bifurcation of the precapillary arterioles, and Friedenwald therefore concluded that these lesions were ischaemic infarcts. ${ }^{2}$

Two technical developments since 1949 have made it easier to study cotton-wool spots. In $1960 \mathrm{~T}$. Kuwabara and D. G. Cogan discovered that the entire retinal vasculature

\footnotetext{
1 Liebreich, R. L., Ophthalmoskopischer Befund bei Morbus Brightii. Arch. f. Ophthalmologie, 5 (part 2), p. 265, 1859.

: Friendenwald, J. S., Amer. f. Ophthal., 1949, 32, 487

- Kuwabara, T., and Cogan, D. G., Arch. Ophth., 1960, 64, 904.

Gay, A. J., Goldor, H., and Smith, M.. Invest. Ophth., 1964, 3, 647.

Ashton, N., and Henkind, P., Brit. F. Oph h., 1965, 49. 225.

- and Harry, J., Trans. Öphthal. Soc. U.K., 1963, 83, 91.

Dodlery, C. T., Henkind, P., Paterson, J. W. Med., Ramalho, P. S., and Hill, Dollery, C. T., Henkind, P., Paterson, J. W.,

- Shakib, M., and Ashton, N., ibid., 1966, 50, 325.
}

could be detached from the retina after it was incubated in a solution of trypsin. Because conventional stains can be used to stain the vascular network free from non-vascular components which also take up the dyes, a much better visualization of retinal vessels became possible. ${ }^{3}$

The second technical advance was the almost simultaneous discovery in two laboratories of a method of experimental production of cotton-wool spots by intracarotid injection of microspheres of known size. Ranging from 7 to 40 microns in size, these microspheres can be either of latex ${ }^{4}$ or of glass. ${ }^{5}$

In $1963 \mathrm{~N}$. Ashton and J. Harry ${ }^{6}$ used trypsin digestion of a retina from a hypertensive subject to show hyaline fatty changes typical of fibrinoid necrosis in the terminal and precapillary arterioles supplying the cotton-wool-spot-bearing area of the retina. They also showed that the capillaries at the site of the cotton-wool spot, though obliterated, were not destroyed and that the capillary bed reopens as the lesion resolves. They ascribed the arteriolar changes to endothelial injury caused by hypertension.

When studied by fluorescence angiography cotton-wool spots show two constant features: there is failure of capillary filling during the angiographic phase, and profuse fluorescein leakage for a long period afterwards. This last phenomenon led J. V. Hodge and C. T. Dollery ${ }^{7}$ to postulate that cottonwool spots were caused by an exudative reaction to arteriolar injury. The pathogenesis of cotton-wool spots was recently re-examined by Dollery and his colleagues ${ }^{8}$ by means of experimental occlusion of retinal arterioles in the pig by the intracarotid injection of a suspension of glass microspheres. Within a few minutes of embolization a grey patch could be observed in the ischaemic region of the retina. This lesion then underwent morphological changes which resulted within one to two days in the development of a cotton-wool spot resembling that of man in both colour and texture. The period of ischaemia necessary to produce a cotton-wool spot need only be short. Thus when within 24 hours the microsphere had moved to a more distal position the whole of the area initially poorly perfused developed a cotton-wool spot.

Since 1950 , in a series of papers issued from his laboratory at the Institute of Ophthalmology, Ashton and his collaborators have made important contributions to our basic knowledge of the pathology of the retinal circulation. In a recent paper M. Shakib and Ashton ${ }^{9}$ have given an account of their study of the ultra-structural changes resulting in the formation of the cotton-wool spot. Within one hour of embolization examination by electron microscopy showed that at the centre of the lesion there was swelling of the nerve fibres and of cells of the plexiform and outer nuclear layers. After 24 hours, when dense white areas indistinguishable from cotton-wool spots appeared in the ischaemic region, there was within the swollen segments of the axons a striking accumulation of mitochondria, neurofilaments, denje bodies, and inclusion membranous whorls. Such a proliferation of axonal organelles could take place only if the ganglion cells of the injured axons had survived. No such changes in the axons were observed in experimental total retinal ischaemia. The cotton-wool spot therefore represents a focal reaction of injured axons of living nerve cells.

The pseudonucleus of the cytoid body almost certainly consists of the conglomeration of these proliferating and degenerating intracellular ultra-structures. Either the swollen axons, or the cytoplasm of the macrophage which has ingested them, account for the pseudocytoplasm of the cytoid body. These observations should therefore put an end to the century-old controversy on the nature of the cytoid body. 